\title{
Assessing the anti-inflammatory effects of quercetin using network pharmacology and in vitro experiments
}

\author{
JINGWEN ZHANG, HONGYAN LI, WEI WANG and HONG LI
}

Department of Endocrinology, Longhua Hospital,

Shanghai University of Traditional Chinese Medicine, Shanghai 200030, P.R. China

Received August 2, 2021; Accepted December 10, 2021

DOI: $10.3892 / \mathrm{etm} .2022 .11230$

\begin{abstract}
The present study aimed to investigate the anti-inflammatory effects of quercetin and the associated mechanisms involved. ELISA, reverse transcription-quantitative PCR and western blot analysis were performed to determine the anti-inflammatory effects of quercetin in lipopolysaccharide (LPS)-stimulated RAW264.7 cells. The molecular mechanisms of quercetin were investigated using network pharmacology, molecular docking technology and in vitro experiments. The results revealed that quercetin reduced the LPS-induced production of TNF- $\alpha$, IL-6 and IL-1 $\beta$ in RAW264.7 macrophages. Protein-protein interaction network topology analysis indicated that Akt was the target of quercetin. Kyoto Encyclopedia of Genes and Genomes analysis indicated that quercetin may regulate the PI3K/Akt signaling pathway to exert its anti-inflammatory effects. Furthermore, the molecular docking results indicated that quercetin had a good affinity for the active sites of Akt. Western blot analysis confirmed that quercetin inhibited the phosphorylation of Akt, with an efficacy stronger than that of an Akt inhibitor. Taken together, Akt served as a target as part of the mechanism of the anti-inflammatory effect of quercetin. This result lays a foundation for the clinical application of quercetin in the treatment of inflammatory diseases.
\end{abstract}

\section{Introduction}

Inflammation is a complex defensive response to several stimuli and injury. According to epidemiological and clinical data, inflammation is a major mechanism underlying chronic diseases or their complications, such as arthritis (1), cardiovascular disease (2), cancer (3) and type II diabetes

Correspondence to: Professor Hong Li, Department of Endocrinology, Longhua Hospital, Shanghai University of Traditional Chinese Medicine, 725 South Wanping Road, Xuhui Area, Shanghai 200030, P.R. China

E-mail: shanhong_li@126.com

Key words: quercetin, inflammation, network pharmacology, molecular docking, experimental validation mellitus (4). Therefore, anti-inflammatory strategies should be adopted in the treatment of these diseases. Currently, the anti-inflammatory drugs approved for clinical use mainly cover non-steroidal anti-inflammatory drugs and corticosteroid hormones. However, the long-term consumption of anti-inflammatory drugs triggers adverse reactions in various organs. Therefore, there is an urgent requirement to discover novel drugs with stronger curative effects and milder side effects.

Quercetin is a type of flavonoid ubiquitous in fruits and vegetables $(5,6)$. This flavonoid has antioxidative, anticancer and anti-inflammatory properties (7). Quercetin inhibits inflammatory responses in numerous diseases; however, the mechanisms involved remain to be clarified $(8,9)$.

Network pharmacology is an analytical tool integrating systems biology, multidirectional pharmacology, computational biology, network analysis and other emerging concepts and methods (10). Network pharmacology has been widely used in the functional analysis of drugs. In the present study, network pharmacology, molecular docking technology and in vitro experiments were used to investigate the molecular mechanisms of the anti-inflammatory effects of quercetin.

\section{Materials and methods}

Analysis of the anti-inflammatory effects of quercetin Materials and reagents. The RAW264.7 cell line was purchased from the Cell Bank of the Chinese Academy of Sciences. DMEM and FBS were from Gibco (Thermo Fisher Scientific, Inc.). Lipopolysaccharide (LPS; cat. no. L2630) was obtained from Sigma-Aldrich (Merck-KGaA). The Cell Counting Kit (CCK)-8 assay kit (cat. no. C0037) was purchased from Beyotime Institute of Biotechnology. The RNA Purification kit (cat. no. B0004DP), RNA reverse transcription (RT) kit (cat. no. EZB-RT2GQ) and the fluorescence quantitative PCR (qPCR) kit (cat. no. A0001-R2) were from EZBioscience. The TNF- $\alpha$ (cat. no. EM3311S), IL-6 (cat. no. EM3201S) and IL-1 $\beta$ (cat. no. EM3184S) ELISA duo set kits were purchased from Biotechwell. Antibodies against phosphorylated (p-)Akt (cat. no. T55561) and Akt (cat. no. T40067) were obtained from Abmart. Antibodies against TNF- $\alpha$ (cat. no. 11948), IL6 (cat. no. 12912), $\beta$-tubulin (cat. no. 2148), GAPDH (cat. no. 5174) and rabbit horseradish peroxidase-conjugated secondary antibodies (cat. no. 7074) were purchased from 
Cell Signaling Technology, Inc. The antibody against IL-1 $\beta$ (cat. no. 26048-1-AP) was obtained from ProteinTech Group, Inc. The Akt inhibitor (cat. no. HY-108232) was purchased from MedChemExpress.

Cell culture. The RAW264.7 cell line was maintained in DMEM containing $10 \% \mathrm{FBS}$ and $100 \mathrm{U} / \mathrm{ml}$ streptomycin/penicillin at $37^{\circ} \mathrm{C}$ in a humidified incubator with $5 \% \mathrm{CO}_{2}$. Cells in the logarithmic phase were used for the experiments.

Cell viability assay. Cell viability was analyzed using the CCK-8 assay according to the manufacturer's protocol. In brief, the cells were seeded into 96 -well plates $\left(1 \times 10^{4}\right.$ cells $\left./ \mathrm{ml}\right)$ for $12 \mathrm{~h}$ and subsequently, quercetin was added to the cells at various concentrations $(0,5,10,20,40$ and $80 \mu \mathrm{M})$, followed by incubation at $37^{\circ} \mathrm{C}$ for $24 \mathrm{~h}$. Subsequently, $10 \mu \mathrm{l}$ CCK- 8 solution was added into each well of the plate and cells were incubated for $3 \mathrm{~h}$ at $37^{\circ} \mathrm{C}$. The optical density was measured at $450 \mathrm{~nm}$ using a microplate reader (BioTek Instruments, Inc.).

$R T$ - $q P C R$. The RAW264.7 cell line was treated with various concentrations of quercetin $(5,10$ and $20 \mu \mathrm{M})$ at $37^{\circ} \mathrm{C}$ for $1 \mathrm{~h}$ in 6-well microplates and stimulated with LPS $(1 \mu \mathrm{g} / \mathrm{ml})$ at $37^{\circ} \mathrm{C}$ for $24 \mathrm{~h}$. The total RNA was extracted according to the manufacturer's specification (EZBioscience). The concentration of RNA was evaluated using spectrophotometric analysis. Total RNA $(1 \mu \mathrm{g})$ was reverse transcribed into cDNA using 4X EZscript Reverse Transcription Mix II. RT was performed at $42^{\circ} \mathrm{C}$ for $15 \mathrm{~min}$. The fluorescence quantitative PCR was performed using a $2 X$ SYBR-Green qPCR Master Mix (ROX2 plus). Relative gene expression levels were obtained following normalization to $\beta$-actin. The following thermocycling conditions were used: Initial denaturation at $95^{\circ} \mathrm{C}$ for $5 \mathrm{~min}$; followed by 40 cycles of $95^{\circ} \mathrm{C}$ for $10 \mathrm{sec}$ and $60^{\circ} \mathrm{C}$ for $30 \mathrm{sec}$ and the reaction was performed in a 7500 Real Time PCR System (Applied Biosystems; Thermo Fisher Scientific, Inc.). The $2^{-\Delta \Delta C q}$ method was used to quantify the results (11). The primer sequences used for qPCR were purchased from Sangon Biotech Co., Ltd. and the sequences of the primers are presented in Table I.

ELISA. Raw 264.7 cells $\left(3 \times 10^{5}\right.$ cells/well) were cultured in 6-well microplates at $37^{\circ} \mathrm{C}$ for $12 \mathrm{~h}$. Thereafter, the RAW264.7 cell line was treated with various concentrations of quercetin $(5,10$ and $20 \mu \mathrm{M})$ at $37^{\circ} \mathrm{C}$ for $1 \mathrm{~h}$ and stimulated with LPS $(1 \mu \mathrm{g} / \mathrm{ml})$ at $37^{\circ} \mathrm{C}$ for $24 \mathrm{~h}$. The cell culture supernatant was then obtained to measure the levels of TNF- $\alpha$, IL- 6 and IL-1 $\beta$ using the ELISA kits according to the manufacturer's protocol.

Western blot analysis. Raw264.7 cells ( $3 \times 10^{5}$ cells/well) were cultured in 6-well microplates at $37^{\circ} \mathrm{C}$ for $12 \mathrm{~h}$. Thereafter, the RAW264.7 cell line was treated with various concentrations of quercetin $(5,10$ and $20 \mu \mathrm{M})$ at $37^{\circ} \mathrm{C}$ for $1 \mathrm{~h}$ and stimulated with LPS $(1 \mu \mathrm{g} / \mathrm{ml})$ at $37^{\circ} \mathrm{C}$ for $6 \mathrm{~h}$. Ice-cold PBS was used to wash the cells three times and lysis solution (cat. no. P0013B; Beyotime Institute of Biotechnology) was used to dissociate the cells on ice for $30 \mathrm{~min}$. The protein concentration was determined using the bicinchoninic acid method. Total protein $(20 \mu \mathrm{g})$ was separated using $10 \%$ SDS-PAGE and then transferred onto polyvinylidene difluoride membranes (cat. no. IPVH00010 PORE; Merck Millipore) for $1 \mathrm{~h}$ at $350 \mathrm{~mA}$. After blocking with 5\% bovine serum albumin (BSA; cat. no. 4240GR100; BioFroxx) for $1 \mathrm{~h}$, the protein was incubated with the primary antibodies [p-Akt $(1: 1,000$ dilution), Akt (1:1,000 dilution), TNF- $\alpha$ (1:500), IL6 (1:500), IL-1 $\beta$ (1:500), $\beta$-tubulin (1:1,000 dilution) or GAPDH (1:1,000 dilution)] overnight at $4^{\circ} \mathrm{C}$. The membranes were washed four times with Tris-buffered saline-Tween-20 (TBST) solution for $10 \mathrm{~min}$ and then incubated with rabbit horseradish peroxidase-conjugated secondary antibodies (1:10,000 dilution) in the presence of BSA for $1 \mathrm{~h}$ at room temperature. Subsequently, the membranes were washed four times with TBST for $10 \mathrm{~min}$. ImageJ software 1.46 (National Institutes of Health) was used for grey value analysis.

\section{Network pharmacological analysis}

Data collection. The targets of quercetin were obtained from the Traditional Chinese Medicine database system pharmacology (TCMSP) and analysis platform (http://tcmspw. com/tcmsp.php) using the key word 'quercetin'. The 'Canonical SMILES' of quercetin was searched using the PubChem database (https://pubchem.ncbi.nlm.nih.gov/) with the key word of 'quercetin' and then imported into Swiss Target Prediction (http://www.swisstargetprediction.ch/index.php) to obtain the targets of quercetin. The targets screened from the two databases were combined and the overlapped targets were removed. Subsequently, the targets associated with inflammation were obtained from the GeneCards database (https://www. genecards.org/), with 'inflammation' as the key word. The targets with a relevance score greater than the median digit were screened.

Protein-protein interaction (PPI) network and topology analysis. The targets of quercetin were mapped to those of inflammation. The overlapped targets were imported into the Search Tool for the Retrieval of Interacting Genes/proteins database (https://string-db.org/) to construct the PPI network. The host species was limited to 'Homo sapiens' and the confidence score for the correlation degree was set to $\geq 0.4$. Subsequently, Cytoscape v3.7.2 was used for topology analysis.

Enrichment analyses. Gene set enrichment analysis was performed using the Database for Annotation, Visualization and Integrated Discovery (DAVID; https://david.ncifcrf. gov/). Gene Ontology (GO) and Kyoto Encyclopedia Genes and Genomes enrichment (KEGG) analyses were performed using the DAVID v6.7 database. The species was set as 'Homo sapiens' and the significance threshold was set as $\mathrm{P}<0.05$.

Molecular docking. The molecular docking analysis was performed using the TCM Network Pharmacology Analysis System (TCMNPAS; no. 2019SR1127090; http://54.223.75.62:3838/npa4/). The PSOVina algorithm was used for molecular docking. First, the 'Canonical SMILES' of quercetin was searched using the PubChem database and the 'protein database (PDB) ID' of Akt (3096) was obtained from the PDB database (https://www.rcsb.org/). Subsequently, the 'Canonical SMILES' of quercetin and 'PDB ID' of Akt were added to the molecular docking module of TCMNPAS. 
Table I. Primers used for quantitative PCR.

\begin{tabular}{|c|c|c|c|c|}
\hline Gene & $\begin{array}{c}\text { GenBank } \\
\text { accession no. }\end{array}$ & Direction & Sequence $\left(5^{\prime}-3^{\prime}\right)$ & $\begin{array}{l}\text { Product } \\
\text { length (bp) }\end{array}$ \\
\hline \multirow[t]{2}{*}{$\beta$-actin } & \multirow[t]{2}{*}{ NM_007393.5 } & Forward & ACTGTCGAGTCGCGTCC & 17 \\
\hline & & Reverse & CCCACGATGGAGGGGAATAC & 20 \\
\hline \multirow[t]{2}{*}{ TNF- $\alpha$} & \multirow[t]{2}{*}{ NM_001278601.1 } & Forward & CCCTCCAGAAAAGACACCATG & 21 \\
\hline & & Reverse & CACCCCGAAGTTCAGTAGACAG & 22 \\
\hline \multirow[t]{2}{*}{ IL-6 } & \multirow[t]{2}{*}{ NM_001314054.1 } & Forward & GGGACTGATGCTGGTGACAAC & 21 \\
\hline & & Reverse & СААСТСТTTTCTCATTTCCACGA & 23 \\
\hline \multirow[t]{2}{*}{ IL-1 $\beta$} & \multirow[t]{2}{*}{ XM_006498795.5 } & Forward & GCTTCAGGCAGGCAGTATCA & 20 \\
\hline & & Reverse & TGCAGTTGCTAATGGGAACG & 20 \\
\hline
\end{tabular}

'From ligand' is an online method to obtain protein docking pocket parameters based on the ligand position. The 'From ligand' button was then selected. Finally, the conformation and docking results of the compounds were downloaded and imported into Pymol3.8 (https://pymol.org) to visualize their three-dimensional structure.

\section{In vitro experiments}

Effects of quercetin on the phosphorylation of Akt. The RAW264.7 cell line was treated with various concentrations of quercetin $(5,10$ or $20 \mu \mathrm{M})$ at $37^{\circ} \mathrm{C}$ for $1 \mathrm{~h}$ in 6 -well microplates and stimulated with LPS $(1 \mu \mathrm{g} / \mathrm{ml})$ at $37^{\circ} \mathrm{C}$ for $6 \mathrm{~h}$. Other experimental steps were mentioned previously in the western blot analysis subsection.

Comparison of the efficacy of the Akt inhibitor MK-2206 and quercetin. The RAW264.7 cell line was preincubated with quercetin $(20 \mu \mathrm{M})$ and the AKT inhibitor (MK-2206; $5 \mu \mathrm{M})$ at $37^{\circ} \mathrm{C}$ for $1 \mathrm{~h}$ in 6 -well microplates and stimulated with LPS $(1 \mu \mathrm{g} / \mathrm{ml})$ at $37^{\circ} \mathrm{C}$ for 6 or $24 \mathrm{~h}$. Other experimental steps were mentioned previously in the RT-qPCR and western blot analysis subsections.

Statistical analysis. All the experiments were performed at least 3 times. Data in this study were statistically processed by SPSS Statistics 22.0 (IBM Corporation).Values are expressed as the mean \pm standard deviation. Significant differences were analyzed using one-way ANOVA followed by Tukey's post-hoc test. $\mathrm{P}<0.05$ was considered to indicate a statistically significant difference.

\section{Results}

Anti-inflammatory effects of quercetin

Effects of quercetin on cell viability. The results of the CCK-8 assay indicated that treatment with quercetin $(5-20 \mu \mathrm{M})$ for $24 \mathrm{~h}$ exerted no notable effects on cell viability (Fig. 1). Therefore, 5-20 $\mu \mathrm{M}$ quercetin was used for the subsequent experiments.

Effects of quercetin on TNF- $\alpha, I L-6$ and $I L-1 \beta$. As presented in Fig. 2A, after treatment with LPS, the mRNA expression levels of TNF- $\alpha$, IL- 6 and IL- $1 \beta$ were significantly increased compared with those in the blank group. In comparison,

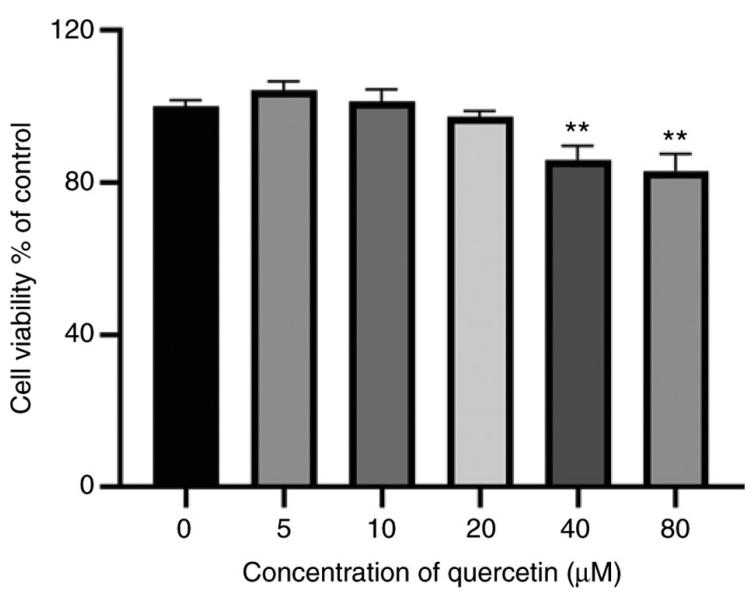

Figure 1. Effects of quercetin on the viability of the RAW264.7 cell line. ${ }^{* *} \mathrm{P}<0.01$ vs. control.

pre-treatment with quercetin decreased the mRNA expression levels of TNF- $\alpha$, IL- 6 and IL-1 $\beta$ in the RAW264.7 cell line in a dose-dependent manner.

The results of the ELISA suggested that after stimulation with LPS, the concentrations of TNF- $\alpha$, IL- 6 and IL- $1 \beta$ were significantly increased and this increase was attenuated in the groups pre-treated with quercetin (Fig. 2B).

Western blot analysis also indicated that after stimulation with LPS, the protein expression levels of TNF- $\alpha$, IL-6 and IL-1 $\beta$ were significantly increased. However, in comparison, pre-treatment with quercetin decreased the protein expression levels of TNF- $\alpha$, IL- 6 and IL-1 $\beta$ in the RAW264.7 cell line (Fig. 2C and D).

Results of network pharmacological analysis Targets of quercetin and inflammation. A total of 226 potential targets of quercetin were obtained using the TCMSP and Swiss Target Prediction databases. A total of 10,454 differentially expressed genes were obtained from the GeneCards database and 5,210 targets with a Relevance Score $>0.947288$ were screened. Finally, 188 targets were selected for enrichment analyses from an overlap in the Venn diagram (Fig. 3).

Network construction and topology analysis. Fig. 4A presents the PPI network of the potential targets of quercetin treatment 

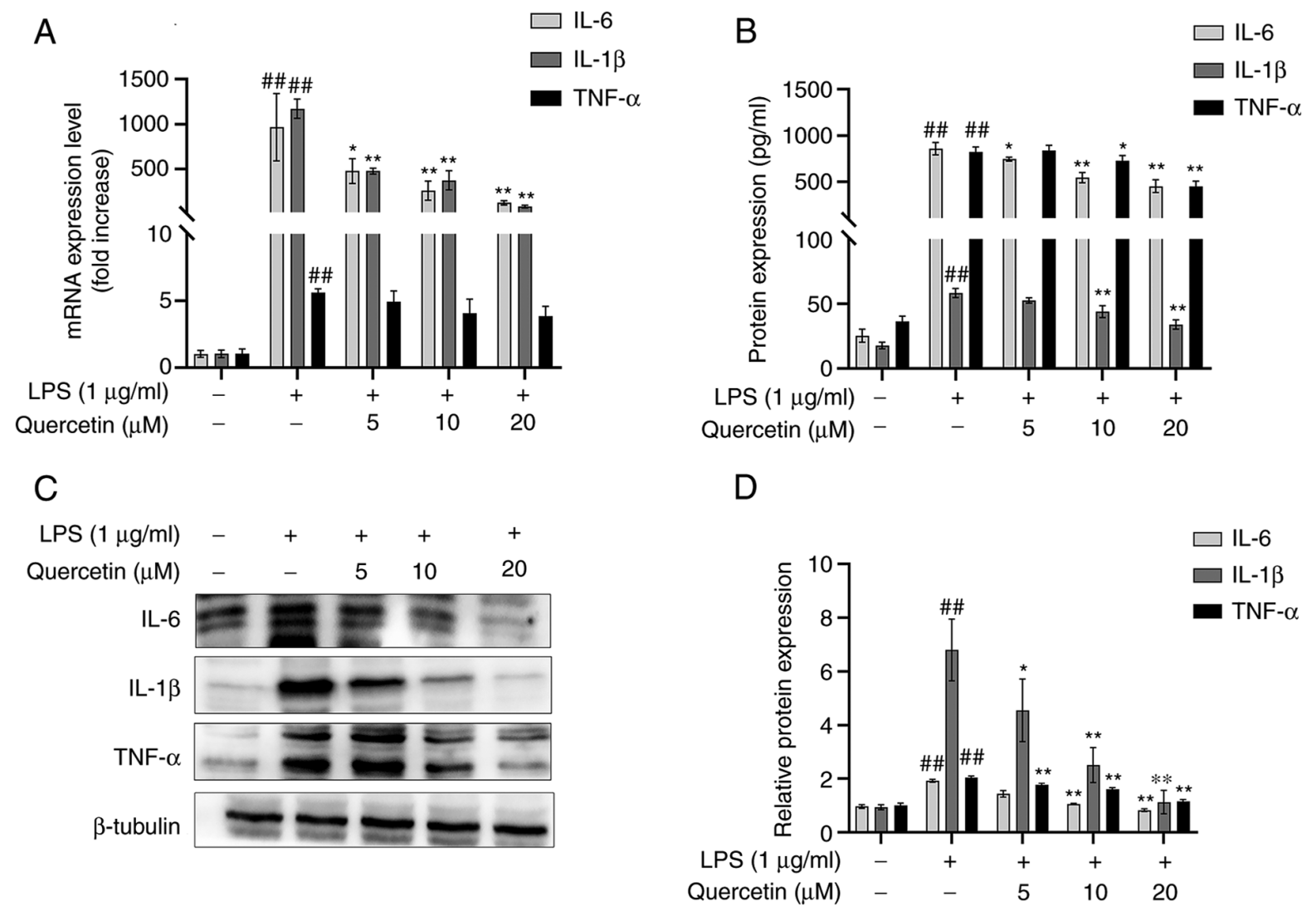

Figure 2. Effects of quercetin on the mRNA expression levels and concentration of TNF- $\alpha$, IL- 6 and IL-1 $\beta$. (A and B) The cells were pretreated with different concentrations of quercetin $(5,10$ and $20 \mu \mathrm{M})$ for $1 \mathrm{~h}$ and then stimulated with LPS $(1 \mu \mathrm{g} / \mathrm{ml})$ for another $24 \mathrm{~h}$. The expression levels of TNF- $\alpha$, IL-6 and IL-1 $\beta$ were analyzed by (A) reverse transcription-quantitative PCR and (B) ELISA. (C and D) The cells were pretreated with different concentrations of quercetin $(5,10$ and $20 \mu \mathrm{M})$ for $1 \mathrm{~h}$ and then stimulated with LPS $(1 \mu \mathrm{g} / \mathrm{ml})$ for another $6 \mathrm{~h}$. The protein expression levels of TNF- $\alpha$, IL- 6 and IL- $1 \beta$ were analyzed using western blot analysis. (C) Representative western blots and (D) quantified results. ${ }^{\# \#} \mathrm{P}<0.01$ vs. non-intervention group; ${ }^{*} \mathrm{P}<0.05$, ${ }^{* *} \mathrm{P}<0.01$ vs. LPS alone group. LPS, lipopolysaccharide.

to counteract inflammation. The PPI network was constructed and visualized using Cystoscape. The nodes and edges are presented in Fig. 4B. All the Akt targets are displayed in the figure. The average node degree value in the PPI network was 34.556. The top 10 nodes with the largest degree of connectivity values were AKT1, TP53, IL6, VEGFA, IL1B, CASP3, JUN, MYC, EGFR and PTGS2.

Enrichment analysis of key targets. A total of 188 selected key targets were submitted for GO and KEGG enrichment analyses and screened with a cutoff of $\mathrm{P}<0.05$. A total of 651 targets were identified in the category biological process (BP), 65 in cellular component (CC) and 144 in the category molecular function (MF), while there were 122 enriched KEGG pathways. The top 10 enriched GO and KEGG pathway terms are presented in Fig. 5. The BP targets included positive regulation of transcription from RNA polymerase II promoter, signal transduction and negative regulation of apoptotic process. The CC targets included nucleus, cytoplasm and cytosol. The MF targets included protein binding, ATP blinding and identical protein binding. The KEGG pathways included cancer, PI3K/AKT signaling pathway and the Hepatitis B signaling pathway.

Molecular docking analysis. Molecular docking analysis was performed to estimate the binding between quercetin and

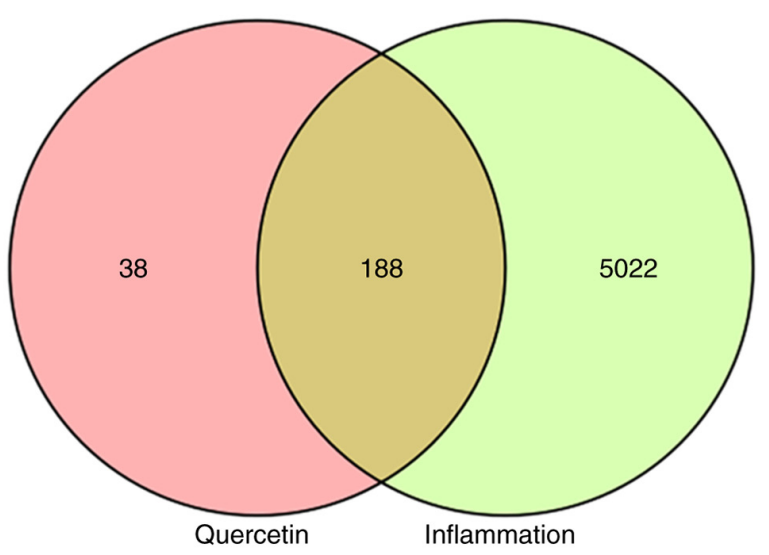

Figure 3. Potential targets of quercetin in the treatment of inflammation. The 226 potential targets of the quercetin were mapped to the 5,210 targets of inflammation. A total of 188 potential targets for Gene Ontology analysis were obtained.

Akt1. The results indicated that the binding force of quercetin with Akt1 was-8.9 kJ/mol, indicating that they had a good affinity. The three-dimensional structure of the molecular docking diagram is provided in Fig. 6.

Experimental verification. As presented in Fig. 7A and B, quercetin dose-dependently inhibited the phosphorylation of 


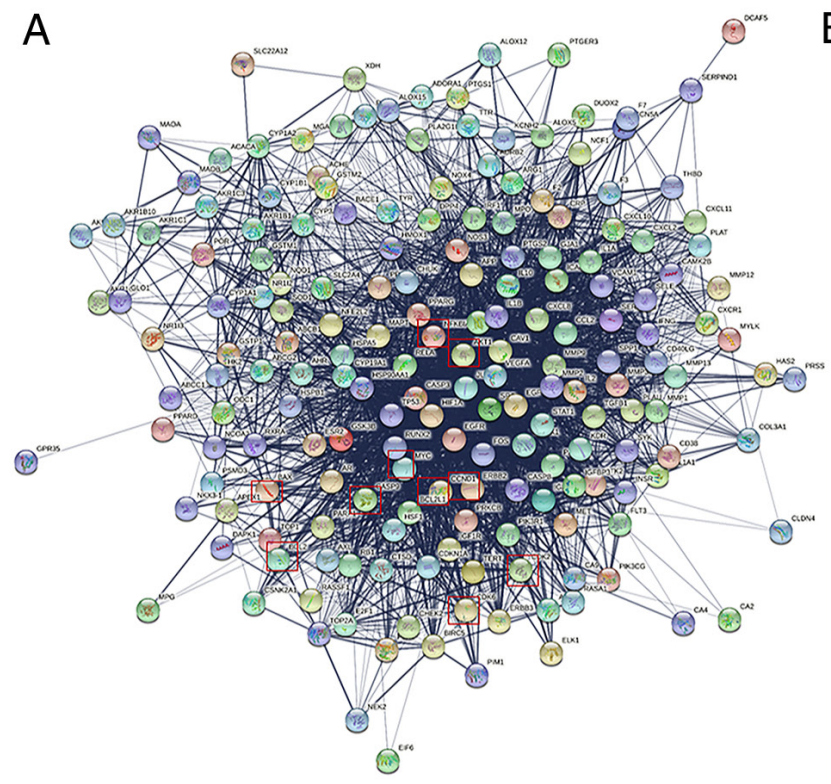

\section{B}

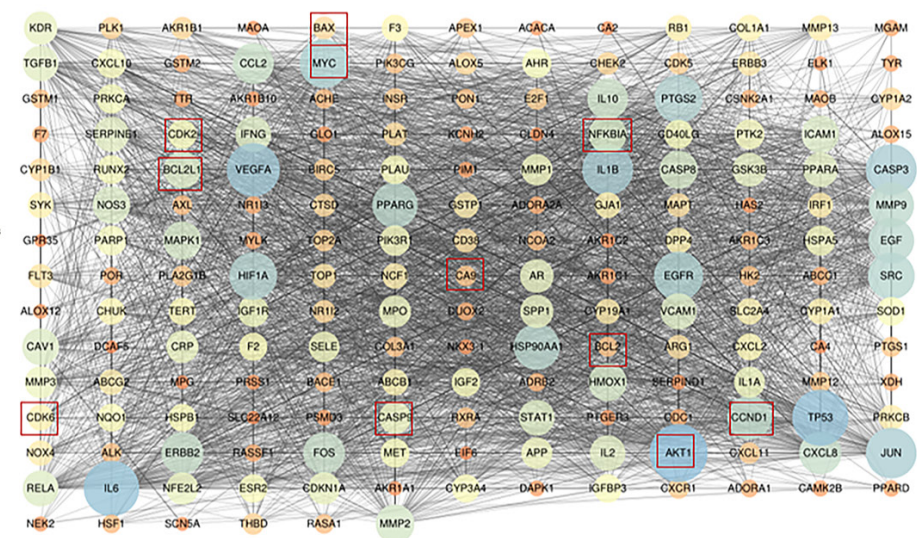

Figure 4. Network construction and topology analysis. (A) PPI network of the potential targets of quercetin in the treatment of inflammation. (B) The visualized PPI network (the size and color of the nodes represent the degree value. The larger the node, the larger the degree value. The width of edges is used to represent the size of EdgeBetweenness values. The thicker the edge is, the larger the value). The red frames indicate all the targets of AKT. PPI, protein-protein interaction.
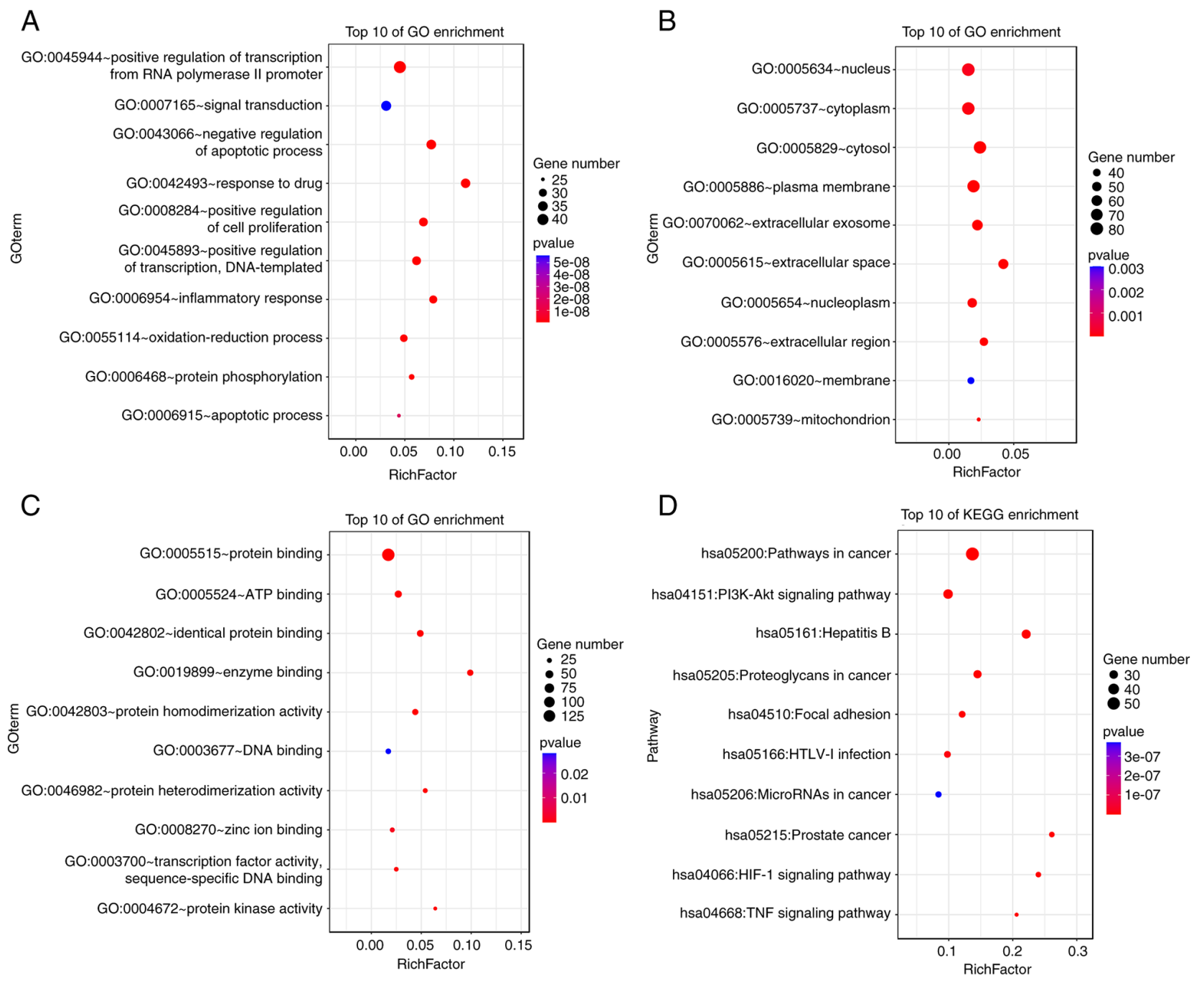

Figure 5. Enrichment analysis of key targets. GO functional terms in the categories (A) biological process, (B) cellular component and (C) molecular function. (D) KEGG pathway enrichment analysis. The top 10 terms in each category are provided. KEGG, Kyoto Encyclopedia of Genes and Genomes. 


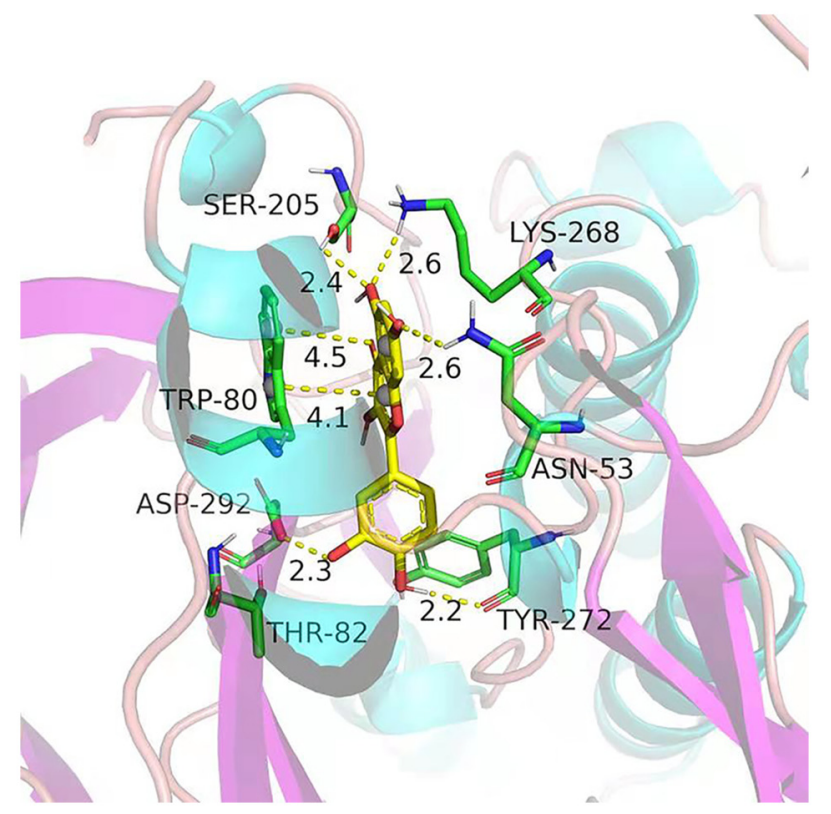

Figure 6. Molecular docking analysis A three-dimensional model of the binding pattern of quercetin at the active site of the protein Akt (binding energy, $-8.9 \mathrm{~kJ} / \mathrm{mol})$. Active-site amino acid residues are represented as tubes, while the compound is presented using a stick model.

A

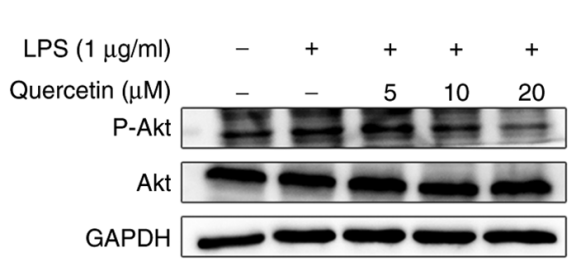

D

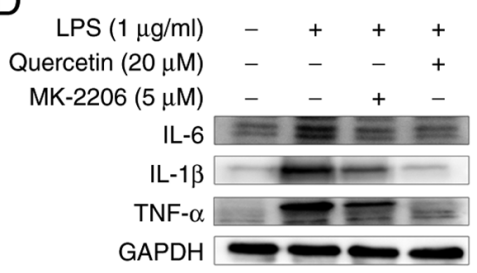

B

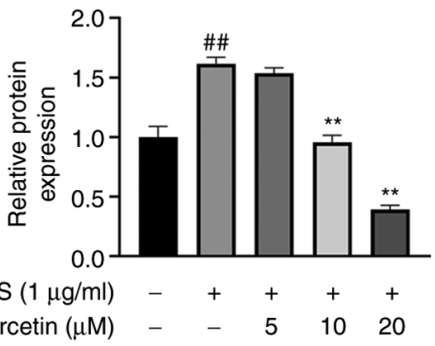

$\mathrm{E}$

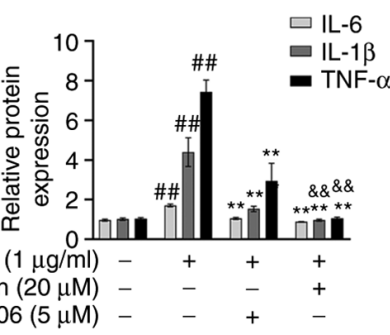

G

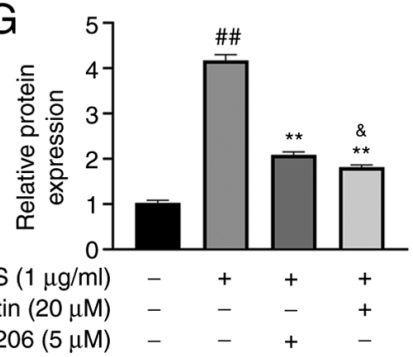

C

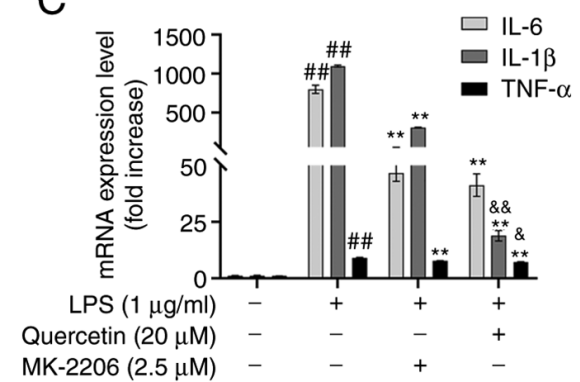

$\mathrm{F}$

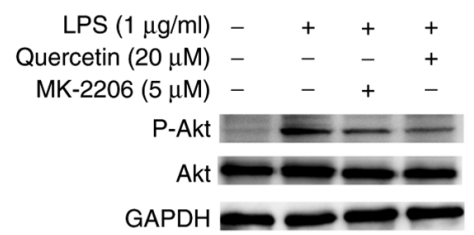

Figure 7. Effects of quercetin on the phosphorylation of Akt in LPS-stimulated RAW264.7 cells. (A and B) The cells were pretreated with different concentrations of quercetin $(5,10$ and $20 \mu \mathrm{M})$ for $1 \mathrm{~h}$ and then stimulated with LPS $(1 \mu \mathrm{g} / \mathrm{ml})$ for another $6 \mathrm{~h}$. (A) After different treatments, the protein levels of p-Akt, Akt and GAPDH were determined using western blot analysis and (B) the results were statistically analyzed. (C-G) RAW264.7 cells were pretreated with quercetin $(20 \mu \mathrm{M})$ and AKT inhibitor (MK-2206; $5 \mu \mathrm{M})$ for $1 \mathrm{~h}$ in 6 -well microplates and stimulated with LPS (1 $\mu \mathrm{g} / \mathrm{ml})$ for 6 or $24 \mathrm{~h}$. The (C) mRNA and (D and E) protein expression levels of TNF- $\alpha$, IL- 6 and IL-1 $\beta$ were analyzed using reverse transcription-quantitative PCR and western blot analysis, respectively. (D) Representative western blots and (E) quantified results. (F and G) The protein levels of p-Akt, Akt were analyzed using western blot analysis. (F) Representative western blots and (G) quantified results. ${ }^{\# \#} \mathrm{P}<0.01$ vs. non-intervention group; ${ }^{* *} \mathrm{P}<0.01 \mathrm{vs}$. LPS alone group; ${ }^{\&} \mathrm{P}<0.05$, \& \& $\mathrm{P}<0.01 \mathrm{vs.} \mathrm{quercetin}$ treatment group. LPS, lipopolysaccharide; p, phosphorylated. 
Akt, suggesting that quercetin counteracted inflammation by repressing Akt phosphorylation.

As indicated in Fig. 7C-E, MK-2206 and quercetin decreased the expression levels of TNF- $\alpha$, IL- 6 and IL- $1 \beta$, and quercetin had a stronger efficacy than MK-2206.

As presented in Fig. 7F and G, MK-2206 and quercetin inhibited the phosphorylation of Akt and quercetin had a stronger efficacy than MK-2206.

\section{Discussion}

Based on RAW264.7 macrophages, LPS-induced inflammation models may be constructed to analyze the pathogenesis of inflammation-related diseases. In the present study, RT-qPCR, ELISA and western blot analysis indicated that LPS significantly increased the concentration of TNF- $\alpha$, IL- 6 and IL-1 $\beta$. It was also confirmed that quercetin exhibited anti-inflammatory effects by reducing the expression levels of TNF- $\alpha$, IL- 6 and IL-1 $\beta$ in LPS-induced RAW264.7 macrophages. Shen et al (12) indicated that quercetin was able to decrease the secretion of IL- 6 , TNF- $\alpha$ and IL-1 $\beta$ in the serum and ankle joint tissues. In addition, another previous study suggested that quercetin attenuated copper sulfate-induced upregulation of TNF- $\alpha$, IL-6 and IL-1 $\beta$ (13). The aforementioned results are consistent with those observed in the present study, indicating that quercetin has an anti-inflammatory effect.

PPI network topology analysis suggested that Akt was the target of quercetin, with the largest degree of connectivity value in the network. KEGG analysis indicated that the anti-inflammatory effects of quercetin may be mediated via the PI3K-Akt signaling pathway. A binding energy of $>4.25$ is considered fair, $5 \leq$ binding energy $<7$ is considered good and binding energy $\geq 7$ is considered excellent; this scoring is conventionally used to classify ligand binding activity (14). The binding energy of quercetin with Akt1 was- $8.9 \mathrm{~kJ} / \mathrm{mol}$, indicating that they had a good affinity. Taken together, it was hypothesized that Akt may be an important target involved in the mechanisms of the anti-inflammatory action of quercetin. As expected, western blot analysis confirmed that quercetin exerted anti-inflammatory effects by repressing the activity of Akt in vitro.

Akt, also known as protein kinase B, is a major downstream effector of PI3K. Akt is divided into three subtypes (Akt1, Akt2 and Akt3). Previous studies have indicated that Akt is associated with inflammation (15-17). The present study suggested that the anti-inflammatory effects of quercetin may arise from the inactivation of Akt. Gulati et al (18) reported that quercetin inhibited Akt activation in human breast cancer cells, which is consistent with the results of the present study. Akt is widely known to be the downstream target of a vast number of cellular processes $(19,20)$, so it is probable that there are also numerous downstream/indirect pathways through which quercetin affects Akt, not only by direct binding. This is also indicated by the high network connectivity of Akt, which suggests a hub gene function of Akt and its key role/involvement in multiple pathways, supporting that the mechanism of quercetin comprises not just 1 direct binding interaction with Akt.

In conclusion, the present study revealed that Akt may be an important target involved in the anti-inflammatory mechanisms of quercetin. These findings provide a new direction for investigating the anti-inflammatory mechanisms of quercetin.

\section{Acknowledgements}

The authors would like to thank Dr Ming Yang, Office of Good Clinical Practice, Longhua Hospital, Shanghai University of Traditional Chinese Medicine (TCM) (Shanghai, China), who developed the TCM Network Pharmacology Analysis System (TCMNPAS) as a major contributor. The molecular docking analysis of the present study was performed using the TCMNPAS (registration no. 2019SR1127090, http://54.223.75.62:3838/npa4/).

\section{Funding}

This research was supported by grants from the Shanghai Committee of Science and Technology Research Projects (grant nos. 19140904600 and 18401900800) and the 2018-2020 Three-year Action Plan for Traditional Chinese Medicine Further Development in Shanghai (grant no. ZY2018-2020-CCCX-2002-08).

\section{Availability of data and materials}

The datasets used and/or analyzed during the current study are available from the corresponding author on reasonable request.

\section{Authors' contributions}

The authors' responsibilities were as follows: JZ and HongL designed the study; JZ and HongyanL performed the experiments; WW analyzed the data; JZ wrote the manuscript, and HongyanL helped edit the paper; JZ and WW checked and confirmed the authenticity of the raw data; HongL revised the manuscript. All authors read and approved the final manuscript.

\section{Ethics approval and consent to participate}

Not applicable.

\section{Patient consent for publication}

Not applicable.

\section{Competing interests}

The authors declare that they have no competing interests.

\section{References}

1. Noack M and Miossec P: Selected cytokine pathways in rheumatoid arthritis. Semin Immunopathol 39: 365-383, 2017.

2. Poledne R and Lesná IK: Inflammation and atherosclerosis. Vnitr Lek 64: 1142-1146, 2019.

3. Diakos IC, Charles KA, McMillan DC and Clarke SJ: Cancer-related inflammation and treatment effectiveness. Lancet Oncol 15: e493-e503, 2014.

4. Lemmers RFH, van Hoek M, Lieverse AG, Verhoeven AJM, Sijbrands EJG and Mulder MT: The anti-inflammatory function of high-density lipoprotein in type II diabetes: A systematic review. J Clin Lipidol 11: 712-724.e5, 2017. 
5. Williamson G and Manach C: Bioavailability and bioefficacy of polyphenols in humans. II. Review of 93 intervention studies. Am J Clin Nutr 81 (Suppl 1): 243S-255S, 2005

6. Mennen LI, Walker R, Bennetau-Pelissero C and Scalbert A Risks and safety of polyphenol consumption. Am J Clin Nutr 81 (1 Suppl): 326S-329S, 2005.

7. Woodman OL and Chan EC: Vascular and anti-oxidant actions of flavonols and flavones. Clin Exp Pharmacol Physiol 31: 786-790, 2004.

8. Lu S, Zhou S, Chen J, Zheng J, Ren J, Qi P, Zhu Z and Li Z: Quercetin nanoparticle ameliorates lipopolysaccharide-triggered renal inflammatory impairment by regulation of Sirt1/NF- $\kappa \mathrm{B}$ pathway. J Biomed Nanotechnol 17: 230-241, 2021.

9. Li CY, Cheng SE, Wang SH, Wu JY, Hsieh CW, Tsou HK and Tsai MS: The anti-inflammatory effects of the bioactive compounds isolated from alpinia officinarum hance mediated by the suppression of NF-KB and MAPK signaling. Chin $\mathrm{J}$ Physiol 64: 32-42, 2021.

10. Berger SI and Iyengar R: Network analyses in systems pharmacology. Bioinformatics 25: 2466-2472, 2009.

11. Livak KJ and Schmittgen TD: Analysis of relative gene expression data using real-time quantitative PCR and the 2(-Delta Delta C(T)) method. Methods 25: 402-408, 2001.

12. Shen P, Lin W, Ba X, Huang Y, Chen Z, Han L, Qin K, Huang Y and Tu S: Quercetin-mediated SIRT1 activation attenuates collagen-induced mice arthritis. J Ethnopharmacol 279: 114213, 2021.

13. Peng X, Dai C, Zhang $M$ and Das Gupta S: Molecular mechanisms underlying protective role of quercetin on copper sulfate-induced nephrotoxicity in mice. Front Vet Sci 7: 586033, 2021.
14. Hsin K, Ghosh S and Kitano H: Combining machine learning systems and multiple docking simulation packages to improve docking prediction reliability for network pharmacology. PLoS One 8: e83922, 2013.

15. Seshadri VD: Brucine promotes apoptosis in cervical cancer cells (ME-180) via suppression of inflammation and cell proliferation by regulating $\mathrm{PI} 3 \mathrm{~K} / \mathrm{AKT} / \mathrm{mTOR}$ signaling pathway. Environ Toxicol 36: 1841-1847, 2021.

16. Kim MJ, Kim DH, Bang E, Noh SG, Chun P, Yokozawa T, Moon HR and Chung HY: PPAR $\alpha$ Agonist, MHY3200, alleviates renal inflammation during aging via regulating ROS/Akt/FoxO1 signaling. Molecules 26: 3197, 2021.

17. Gao HN, Hu H, Wen PC, Lian S, Xie XL, Song HL, Yang ZN and Ren FZ: Yak milk-derived exosomes alleviate lipopolysaccharide-induced intestinal inflammation by inhibiting PI3K/AKT/C3 pathway activation. J Dairy Sci 104: 8411-8424, 2021.

18. Gulati N, Laudet B, Zohrabian VM, Murali R and JhanwarUniyal M: The antiproliferative effect of quercetin in cancer cells is mediated via inhibition of the PI3K-Akt/PKB pathway. Anticancer Res 26: 1177-1181, 2006.

19. Bellacosa A, Kumar CC, Di Cristofano A and Testa JR: Activation of AKT kinases in cancer: Implications for therapeutic targeting. Adv Cancer Res 94: 29-86, 2005.

20. Martini M, De Santis MC, Braccini L, Gulluni F and Hirsch E: PI3K/AKT signaling pathway and cancer: An updated review. Ann Med 46: 372-383, 2014.

This work is licensed under a Creative Commons Attribution-NonCommercial-NoDerivatives 4.0 International (CC BY-NC-ND 4.0) License. 